\title{
Relative sensitivity of magnetic resonance spectroscopy and quantitative magnetic resonance imaging to cognitive function among nondemented individuals infected with HIV
}

\author{
ROBERT H. PAUL, ${ }^{1}$ THOMAS ERNST, ${ }^{2}$ ADAM M. BRICKMAN, ${ }^{3}$ \\ CONSTANTIN T. YIANNOUTSOS, ${ }^{4}$ DAVID F. TATE, ${ }^{5}$ RONALD A. COHEN, ${ }^{6}$ \\ BRADFORD A. NAVIA, ${ }^{7}$ for the ACTG 301 and 700 teams and the HIV MRS Consortium \\ ${ }^{1}$ Department of Psychology, Behavioral Neuroscience, University of Missouri, St. Louis, St. Louis, Missouri \\ ${ }^{2}$ Department of Medicine, University of Hawaii, Honolulu, Hawaii \\ ${ }^{3}$ Taub Institute for Research on Alzheimer's Disease \& The Aging Brain, College of Physicians and Surgeons, \\ Columbia University, New York, New York \\ ${ }^{4}$ Indiana University School of Medicine, Indianapolis, Indiana \\ ${ }^{5}$ Brigham and Women's Hospital, Center for Neurological Imaging, Harvard Medical School, Boston, Massachusetts \\ ${ }^{6}$ Department of Psychiatry, Brown Medical School, Center for AIDS Research, Providence, Rhode Island \\ ${ }^{7}$ Departments of Neurology and Psychiatry, Tufts-New England Medical Center, Boston, Massachusetts
}

(Received May 17, 2007; Final Revision April 7, 2008; Accepted April 8, 2008)

\begin{abstract}
In the present study, we examined the relationships among cognitive function, magnetic resonance spectroscopy (MRS) brain metabolite indices measured in the basal ganglia, and quantitative magnetic resonance imaging (MRI) of the caudate nucleus and the putamen in the earliest stages of HIV-related cognitive involvement. Participants included $22 \mathrm{HIV}$-positive individuals and $20 \mathrm{HIV}$-negative individuals. HIV-positive individuals performed significantly more poorly than the HIV-negative individuals on several cognitive measures. In addition, the choline/creatine ratio was significantly higher and the $\mathrm{N}$-acetyl aspartate/choline ratio was significantly lower among HIV patients. The caudate and putamen sizes were smaller among HIV-positive patients compared with controls; however, the differences did not reach statistical significance. Correlation analyses revealed associations between cognitive function and select MRS indices. In addition, caudate size was significantly correlated with performances on higher-order thinking tests whereas putamen size was significantly correlated with performances on motor tests. The results suggest that MRS differences are more pronounced than area size differences between seropositive and seronegative individuals in mild stages of HIV-related cognitive impairment. However, basal ganglia size remains an important contributor to cognitive status in this population. Longitudinal studies are needed to determine the evolution of these imaging correlates of HIV-cognitive impairment in HIV.

(JINS, 2008, 14, 725-733.)
\end{abstract}

Keywords: MRS, Neuroimaging, Infection, Circuit, Subcortical, Brain

\section{INTRODUCTION}

Despite the benefits afforded by antiretroviral therapy (ART), the frequency of mild cognitive impairment among infected individuals remains a significant concern for individuals infected with human immunodeficiency virus (HIV). Before

Correspondence and reprint requests to: Robert Paul, University of Missouri, St. Louis, Department of Psychology, One University Blvd., St. Louis, MO 63121-4400. E-mail: paulro@umsl.edu the introduction of ART, cognitive difficulties were most frequently evident among individuals with significantly depressed immune systems (Cohen et al., 2001; Selnes et al., 1995). However, neuropathological data suggest that the frequency of HIV-related encephalopathy may be increasing in the era of ART (Neuenburg et al., 2002).

Understanding the biomarkers associated with cognitive dysfunction among HIV patients remains a pressing concern, especially given the observation that individuals with HIV are now living longer, and cognitive difficulties are 
not entirely ameliorated with medical management of the virus in the periphery (Brew, 2004; Sacktor et al., 1999, 2001). Furthermore, mild cognitive difficulties are associated with important activities of daily living. For example, HIV patients with mild to moderate cognitive difficulties exhibit impairments in shopping, cooking, financial management, driving performance and perceived quality of life (Heaton et al., 2004; Marcotte et al., 2004; Osowiecki et al., 2000). As such, it remains important to define the neural signatures associated with cognitive difficulties in HIV.

Neuroimaging has offered significant insights into the nature and extent of central nervous system (CNS) changes associated with HIV infection, and the relationship between these changes and cognitive function. In particular, brain metabolite abnormalities measured by magnetic resonance spectroscopy (MRS) are highly sensitive to central nervous system involvement associated with HIV. Concentrations of myoisonitol (MI) a marker of glial activity and $\mathrm{N}$-acetylaspartate (NAA) a marker of mature neurons have received the most attention in the research literature (for reviews see Navia, 1997; Navia and Gonzalez, 1997; Tucker et al., 2004). Abnormal levels of these metabolites have been reported among asymptomatic patients (Meyerhoff et al., 1999; Tarasow et al., 2003), HIV patients with mild cognitive difficulties (Chang et al., 2004), and individuals with HIV-dementia (Yiannoutsos et al., 2004). Increased MI and reduced NAA have been identified most consistently in the subcortical regions particularly the basal ganglia and the white matter, with relative sparing of the cortex (Yiannoutsos et al., 2004). Interestingly, there is some evidence that brain metabolite abnormalities improve after initiating treatment with ART (Chang et al., 1999), however, other data suggest that abnormalities may persist in the context of treatment (Chang et al., 2003).

Studies frequently report MRS indices as ratios often controlling for creatine $(\mathrm{Cr})$ and choline (Cho), which are believed to be relatively stable references, though this is not a universally accepted approach. These metabolites represent a general energy metabolite $(\mathrm{Cr})$ and a metabolite associated with cell membrane synthesis (Cho). We recently examined MRS ratios in a cohort of HIV-infected individuals with AIDS Dementia Classification scale scores ranging from 1 to 3 and reported significant relationships between $\mathrm{NAA} / \mathrm{Cr}$ in the frontal white matter and $\mathrm{MI} / \mathrm{Cr}$ in the basal ganglia with measures of gross and fine motor speed (Paul et al., 2007). These findings were interpreted as evidence of reduced neuronal integrity and increased gliosis among HIVinfected patients.

Similar to MRS indices, quantitative MRI metrics also correlate with cognitive dysfunction associated with HIV particularly in the more advanced stages of the disease (for review, see Paul et al., 2002; Tucker et al., 2004). Structural MRI abnormalities associated with HIV have been recognized since the beginning of the HIV epidemic, with cortical atrophy, reductions in caudate volume, and increased white matter hyperintensities commonly reported (Aylward et al., 1993, 1995; Dal Pan et al., 1992; Grant et al., 1987;
Hestad et al., 1993). Of these findings, caudate volume has emerged as a relatively consistent quantitative MRI variable associated with HIV cognitive status, particularly among demented patients. Most studies have found significantly reduced caudate volumes among HIV patients with dementia compared with healthy control subjects and nondemented HIV patients (see Paul et al., 2002). These findings have typically been interpreted as reflecting the impact of viral aggregation in the region of the basal ganglia, and the subsequent interference in cortical-subcortical circuits involved in cognitive operations.

Few studies have been directed at understanding the links between basal ganglia regions other than the caudate and cognitive performance in HIV. Presumably this reflects the well recognized functional significance of the reciprocal connections between the caudate and the dorsolateral prefrontal cortex, a system known to be involved in higherorder thinking and executive function (for review, see Cummings, 1993). However, the putamen represents an additional structure of the basal ganglia, and the putamen has reciprocal connections with the motor cortex (Romanelli et al., 2005). Because reduction in psychomotor speed and speeded fine motor dexterity are common symptoms of HIV, there exists a theoretical rationale to support the involvement of the putamen in the expression of cognitive-motor symptoms associated with HIV. Furthermore, recent work by Moore et al. (2006) indicates that neuropathology within the putamen (among other brain regions) correlates significantly with overall cognitive status among HIV patients. These findings emphasize the importance of examining the putamen using in vivo neuroimaging.

The studies conducted to date have identified the clinical utility of both MRS and quantitative MRI in the study of HIV cognitive involvement. There have been few studies, however, that have examined the contribution of both neuroimaging methods to cognitive status in the era of ART, particularly in the early stages of HIV-related cognitive impairment. The purpose of the present study was to examine the relationships between cognitive performance, MRS and quantitative MRI among nondemented HIV patients receiving ART. We predicted that HIV patients would exhibit significant alterations in brain metabolites measured in the basal ganglia and reduced caudate and putamen sizes compared with seronegative individuals. We also predicted that both MRS and quantitative MRI indices would correlate with cognitive performance among the HIV patients but not the healthy control subjects.

\section{METHODS}

\section{Subjects}

Subjects for this study were selected from a larger AIDS Clinical Trials Group (ACTG)-sponsored trial of memantine on cognitive function (ACTG 301). The parent study included $100 \mathrm{HIV}$-positive individuals and $20 \mathrm{HIV}$-negative individ- 
uals. The present study included a subset of HIV-positive patients, for whom both MRI and MRS data were available for analyses and for whom demographic variables were relatively well matched with seronegative control subjects. Twenty-two individuals infected with HIV and enrolled in an ACTG sponsored trial of memantine on cognitive function (ACTG 301) were included in the present study. Consistent with the parent study, HIV diagnosis was based on serologic testing by ELISA and Western blot. Patients with a psychiatric disorder other than depression were excluded. Individuals that met DSM-IV criteria for substance abuse or alcohol abuse within the 6 months before study enrollment were excluded. Additional exclusionary criteria included developmental disorder, head injury, or neurologic disease. The majority of HIV patients were currently taking ART. All data reported in the present study were collected at the baseline visit, before initiation of memantine.

The patients included 19 males and 3 females and the sample averaged 37.9 (3.9) years of age. The median CD4 cell count was 341 (range $=37-878$ ). The patient group included 6 individuals diagnosed with ADC stage 0 and 16 individuals diagnosed with ADC stage 1 (consistent with mild cognitive impairment). Individuals with ADC stages 2 and 3 were excluded from the study.

Twenty healthy control subjects were included in the present study. The healthy controls were required to meet the same exclusion criteria as the HIV patients. The healthy control subjects included 10 women and 9 men (sex was not recorded for one subject), and the group averaged 34.7 (10.5) years of age. The HIV-positive individuals did not differ from the HIV-negative individuals in terms of the percentages of participants who completed less than 12 years of education, high school, or more than 12 years of education $(p>.05)$.

\section{Procedure}

Participants completed demographic questionnaires, assessment of mood and neuropsychological evaluation in a single testing session. Neuroimaging was subsequently conducted within 2 weeks of the neuropsychological evaluation. All measures were administered and scored according to standard procedures. Participants received financial compensation for participation in the study. Written informed consent was obtained before enrollment. The protocol was approved by local IRBs.

\section{Mood}

All individuals completed the Center for Epidemiologic Studies Depression Scale (CES-D) to quantify the severity of mood disturbance. The CES-D is a 20-item self-report measure of mood that has been used extensively in HIV-related research. Participants were asked to rate their agreement with each question using a Likert Scale $(1=$ rarely to $4=$ most of the time). Total score served as the dependent measure.

\section{Magnetic resonance imaging}

Neuroimaging was conducted using Signa 1.5 Tesla scanners (GE Medical Systems, Milwaukee, WI). Each system operated with system 5.6 or higher and using a standard GE head coil. Data were collected at six sites including Massachusetts General Hospital (MGH; the central site), University of California, Los Angeles (UCLA), University of Pennsylvania, University of Washington, University of Rochester, Mt. Sinai Medical Center, University of Texas, and University of California, San Francisco (UCSF). The imaging study protocol was directed and monitored by the central site $(\mathrm{MGH})$. Initial MRS studies conducted by the consortium revealed high consistency of MRS spectra across the different imaging sites (Lee et al., 2003). T1-weighted sagittal and high-resolution proton-density images were analyzed in this study. The sequence parameters were as follows: $\mathrm{TR} / \mathrm{TE}=16 / 600 ; \mathrm{FOV}=20 \mathrm{~cm}$; Slice thickness $=$ $3 \mathrm{~mm}$; Matrix size $=256 \times 128$, with 1 NEX. In addition, a short echo-time ( $\mathrm{TE}=35 \mathrm{~ms}$ ) point-resolved spectroscopy (PRESS) sequence was used for MRS data acquisition. The voxel size was $2.0 \times 2.0 \times 1.5 \mathrm{~cm}^{3}$.

\section{$M R S$}

As described in detail previously (Lee et al., 2003), spectra data were sampled in the basal ganglia with the voxel placed in the putamen. Voxel placement verification was determined at the central processing site. We examined five MRS variables obtained from the basal ganglia including $\mathrm{MI} / \mathrm{Cr}$, $\mathrm{NAA} / \mathrm{Cr}$, Cho/Cr, NAA/Cho, and NAA/MI. These ratios have been identified in previous studies as sensitive markers of neuronal injury in HIV (Lee et al., 2003). For this study, we restricted our focus to the basal ganglia to retain consistency with the quantitative MRI analyses. Raw data were analyzed using a standardized protocol including a commercial package (SageIDL; GE Medical Systems). Images and raw spectroscopic data were transferred electronically to $\mathrm{MGH}$ for processing. All data were analyzed using the commercial software package SageIDL (GE Medical Systems).

\section{Quantitative MRI}

We examined caudate and putamen size by means of manual tracing using NIH Image. For each nucleus, two slices were selected, which represented the best anatomic images of the caudate using the Matsui and Hirano atlas according to a protocol described in detail elsewhere (Brickman et al., 2003). Briefly, the left and right nuclei were traced twice and the average of the measures was determined for each slice. The two slices were then averaged to define an average area. All tracing was completed by a highly experienced neuromorphometrist who was blind to group status. The dependent measures included area sizes for both nuclei. Because we selected the average of two slices rather than summed areas of multiple slices, the final dependent variable is identified as area size rather than volume. 


\section{Neuropsychological assessment}

Neuropsychological assessment was conducted by trained research assistants at each site. The cognitive test battery contained measures that have been demonstrated to be sensitive to HIV-related cognitive changes (Miller et al., 1990; Sacktor et al., 2001). The individual measures are described below.

Rey Auditory Verbal Learning Test (RAVLT; Rey, 1964): The RAVLT was administered and scored according to standard methods. Specifically, participants were read a list of words on five trials and were required to recall as many words as possible on each trial. Following a brief delay filled with a distractor list, participants were required to recall as many words as possible from memory. The dependent measures were the total number of words recalled across the five learning trials and the total number of words recalled on the delay trial.

Timed Gait: Participants were instructed to walk as quickly as possible for a distance of 10 meters. Three trials were conducted and the dependent measure was the average time for the three trials.

Grooved Pegboard (Klove, 1963): Participants completed the Grooved Pegboard task using the dominant and nondominant hands on separate trials. All participants were instructed to place the pegs into the grooved holes one-attime as quickly as possible using the designated hand. Time to completion for each hand was the dependent variable. To facilitate data reduction we included only the nondominant hand as the final dependent variable for this study.

Stroop Color Word Interference Test (Comalli et al., 1962): Participants were administered the Comalli modification of the Stroop Test (Stroop, 1935). The test consisted of three trials administered on three cards. On the first trial, participants were instructed to read aloud color words (e.g., blue) printed in black ink. On the second trial participants were presented with columns of blocks printed in colored ink, and they were required to name the colors that the blocks were printed in. On the final trial, participants were presented with color words printed in incongruent ink and they were instructed to name the color that the words were printed in. The dependent variable was time to complete each trial one and trial 3. These two indices provide measures of processing speed and cognitive flexibility respectively.

Symbol Digit Task (Smith, 1973): Participants were instructed to verbally substitute symbols with numbers using a key held in constant view. Total correct within 90 seconds was the dependent variable.

Trail Making (Anonymous, 1944): Consistent with standard administration, participants completed Trail Making A and subsequently completed Trail Making B. On the former task, participants were required to sequentially connect circled numbers using a pencil (e.g., 1-2-3-4 to 25). On the latter task, participants were required to connect in ascending and alternating order, numbers and letters (e.g., 1-A$2-\mathrm{B}$, etc). Time to completion was the dependent variable on each task.
CalCap (Miller, 1990; Miller et al., 1991): The California Computerized Assessment Package (CalCap) is a battery of attention and psychomotor tasks administered using a computer. Two tests from the CalCap were recorded in the parent study, including a test of simple reaction time and a test of sequential reaction time. The test of simple reaction time required participants to depress a key as soon as any stimulus appeared on the computer screen. On the test of sequential reaction time, participants were instructed to depress the key when two numbers appear in sequential order (e.g., the number 5 followed immediately by the number 5). The dependent variable for each test was the median reaction time for correct responses.

\section{Statistical analyses}

Given the difference in male/female distribution between the two groups, we conducted preliminary analyses among the control sample to determine whether there were any significant differences in MRI and MRS indices between males and females. Results of independent $t$ tests revealed no statistically significant group differences in this group. As such, sex was not entered as a covariate in subsequent analyses. A single MANOVA was conducted to examine the neuropsychological measures between groups. The neuropsychological measures included total correct on verbal learning and delayed recall, average timed gait, time to completion for grooved pegboard (nondominant hand), Trail Making A and B, Symbol Digit Test, and the incongruent trial from the Comalli Stroop and simple reaction time and sequential reaction time on the CalCap. Separate analyses were conducted for the MRS and MRI imaging variables, though all dependent measures for each modality were included in each analysis to minimize spurious findings. Univariate analyses were completed when the omnibus MANOVA was statistically significant. Correlations were conducted separately for each group to examine the degree of variance shared between imaging variables and cognitive performance.

\section{Results}

The two groups did not differ significantly in age $[t(39)=$ $-1.8 ; p>.05]$. The HIV-positive individuals reported significantly more symptoms of depression compared with the control subjects $[t(37)=-4.1) ; p<.01]$, however, the average CES-D score was 16, which falls just at the cutoff for clinically meaningful depression. To determine whether depression scores should be included as a covariate in subsequent analyses we conducted Pearson correlation analyses between CES-D scores and all imaging and neuropsychological variables among the HIV-positive cohort. Results revealed no significant associations between depression score and imaging variables, and only one significant relationship between depression score and neuropsychological performance (Trails B, $r=0.55 ; p=.010$ ). Given the lack of consistent relationships between these variables we did not include depression as a covariate in the group comparisons. 


\section{Neuropsychological Assessment of Psychomotor Function and Attention}

A MANOVA was conducted to examine potential group differences on measures of cognitive function (Table 1). The omnibus MANOVA was significant between the groups $[F(11,27)=2.5$, Wilks' lambda $=.49 ; p=.021]$. Univariate contrasts revealed that the HIV-positive individuals performed significantly more poorly than the HIV-negative individuals on Symbol Digit, Grooved pegboard nondominant hand, trials 1 and 3 of the Stroop, total recall and delayed recall of the verbal memory test, both measures of the CalCap test and timed gait. Group performance did not differ on Trail Making A or Trail Making B tests.

\section{MRS Brain Metabolite Levels}

The overall MANOVA comparing brain metabolites between groups was significant (Wilks' lambda $=.69(5,29)=2.5$; $p=.48)$. Univariate contrasts revealed that HIV patients had significantly higher levels of Cho/ $\mathrm{Cr}(p=.005)$ and significantly lower levels of NAA/Cho $(p<.004)$ compared with the healthy control subjects (Table 2). The groups did not differ on the levels of NAA/Cr, MI/Cr, or NAA/MI in the basal ganglia $(p s>.05)$.

\section{Quantitative MRI}

The overall MANOVA for the area sizes was not statistically significant. As evident from Table 2, the mean sizes of the caudate and putamen were lower for the HIV-positive individuals compared with the HIV-negative individuals, but the differences did not reach statistical significance.

\section{Disease Factors}

Neither plasma CD4 cell count nor plasma HIV RNA correlated significantly with putamen or caudate sizes or cognitive performances. However, plasma RNA correlated

Table 1. Neurocognitive performances: Mean $(S D)$

\begin{tabular}{lcc}
\hline \hline Measure & HIV patients & Healthy controls \\
\hline Timed gait & $10.7(2.5)$ & $9.4(1.2)^{*}$ \\
Symbol Digit & $48.8(10.4)$ & $60.7(12.0)^{*}$ \\
Trail Making A & $29.0(10.6)$ & $28.0(13.7)$ \\
Simple reaction time/ms & $462.6(118.0)$ & $389.8(46.1)^{*}$ \\
Choice reaction time/ms & $605.4(113.9)$ & $507.2(78.0)^{*}$ \\
Pegboard-dominant hand & $69.3(11.2)$ & $58.2(7.9)^{*}$ \\
Stroop trial 1 & $73.3(20.5)$ & $57.6(10.3)^{*}$ \\
Trail Making B & $77.3(34.9)$ & $58.3(27.8)$ \\
Stroop interference & $134.2(39.7)$ & $102.3(21.5)^{*}$ \\
Rey verbal learning & $46.3(9.6)$ & $55.2(7.1)^{*}$ \\
Rey delayed recall & $8.8(3.7)$ & $12.3(2.2)^{*}$ \\
\hline \hline
\end{tabular}

$* p<.05$.
Table 2. Quantitative MRI indices and MRS metabolic concentrations: Mean $(S D)$

\begin{tabular}{lcc}
\hline \hline Measure & $\begin{array}{c}\text { HIV-positive } \\
\text { individuals }\end{array}$ & \multicolumn{2}{c}{$\begin{array}{c}\text { HIV-negative } \\
\text { individuals }\end{array}$} \\
\hline Total putamen & $1421.5(212.1)$ & $1446.8(193.7)$ \\
Total caudate & $731.0(106.6)$ & $764.2(78.8)$ \\
NAA/Cr & $1.4(.22)$ & $1.3(.12)$ \\
Cho/Cr & $.84(.19)$ & $.70(.05)^{*}$ \\
NAA/Cho & $1.7(.26)$ & $1.9(.17)^{*}$ \\
NAA/MI & $2.8(.74)$ & $3.0(.55)$ \\
MI/Cr & $.52(.12)$ & $.47(.07)$ \\
\hline \hline
\end{tabular}

$* p<.05$.

strongly with $\mathrm{MI} / \mathrm{Cr}(r=0.68)$ and NAA/MI $(r=-0.59)$ in the basal ganglia.

\section{MRS, MRI, and Cognitive Correlational Analyses}

Correlational analyses of data from the HIV-positive group revealed that specific brain metabolite levels were associated with aspects of cognitive performance. MI/Cr was correlated with Trail Making A $(r=.36 ; p=.14)$, choice reaction time $(r=0.42 ; p=.08)$, timed gait $(r=0.43 ; p=$ $.07)$ but the relationship met criteria for statistical significance only on Grooved Pegboard nondominant ( $r=0.59$; $p=.010)$. NAA/MI was significantly associated with Grooved Pegboard nondominant hand $(r=0.59 ; p=.009)$ and a trend was noted on trail making $\mathrm{B}(r=0.33 ; p=.17)$. NAA/Cho was correlated with performance on nondominant Grooved Pegboard ( $r=-0.63 ; p=.002)$. NAA $/ \mathrm{Cr}$ was not significantly related to the cognitive measures. By comparison, analyses of the healthy controls revealed that only NAA/Cho associated with cognitive performances, with inverse relationships identified on Trail Making A ( $r=$ $-0.63 ; p=.034)$, Trail Making B $(r=-0.63 ; p=.004)$, and Sequential Reaction Time $(r=-0.62 ; p=.006)$.

Relationships between MRI indices and cognition were also significant for the HIV-positive group. Caudate area size correlated significantly with Trail Making B $(r=-0.51$; $p=.015)$, total learning across trials $(r=0.52 ; p=.009)$, trial 1 of the Stroop $(r=0 .-61 ; p=.003)$ timed gait $(r=$ $-0.43 ; p=.04$ ) and trial 3 of the Stroop (interference; $r=$ $-0.52 ; p=.015)$. Putamen size correlated significantly with Symbol digit $(r=0.42 ; p=.046)$ and Grooved Pegboard dominant hand $(r=-0.44 ; p=.042)$. Among the healthy controls, caudate size correlated with total learning $(r=0.53 ; p=.02)$, and putamen size correlated with Trail Making A $(r=-0.71 ; p=.001)$, Trail Making B $(r=$ $-0.54 ; p=.016)$, and Sequential Reaction Time $(r=-0.46$; $p=.045)$. Putamen area size correlated significantly with $\mathrm{NAA} / \mathrm{Cr}$ in the basal ganglia $(r=.44 ; p=.04)$, but no other correlations between MRI and MRS indices were statistically significant. 


\section{DISCUSSION}

Results from the current study provide several important contributions to the current literature. First, select MRS indices differed significantly between nondemented HIVpositive individuals on ART and demographically similar seronegative control subjects. Second, both MRS and quantitative MRI indices were strongly related to cognitive function among the HIV patients. Collectively, the results suggest that metabolite changes may be more pronounced than surface area changes among patients without dementia. However, some individuals may develop quantitative MRI changes relatively early in the course of CNS disturbance and these quantitative MRI changes correlate strongly with specific cognitive functions.

The first observation that MRS abnormalities differed significantly among nondemented individuals is consistent with previous studies demonstrating the sensitivity of MRS to CNS involvement in HIV. There is now a rich literature describing significant brain metabolite changes associated with HIV (for review, see Navia \& Gonzalez, 1997; Navia $\&$ Rostasy, 2005; Tucker et al., 2004). Significantly increased levels of the purported glial markers MI and Cho are consistently observed in the subcortical brain regions, while reductions in the neuronal marker NAA have been identified in the frontal white matter and subcortical grey matter. In addition, MRS metabolite levels correlate with performance on select measures of cognitive function, including tests of fine motor dexterity, executive function, and information processing speed (Chang et al., 1999; Meyerhoff et al., 1999; Yiannoutsos et al., 2004). Cloak and colleagues (2004) have also recently demonstrated that select MRS metabolites map onto changes in the microstructural integrity of the brain in HIV as measured by diffusion tensor imaging (DTI).

It is notable that the majority of patients in the present study were taking ART, and as noted already studies have reported improved cognitive function among HIV patients following treatment with ART (Cohen et al., 2001; Robertson et al., 2004). Evidence that MRS abnormalities persist despite treatment with ART suggests that the CNS compartment remains vulnerable in the context of long-term treatment. This is consistent with the results of an early small study of patients before and after ART (Wilkinson et al., 1997). In this study, patients exhibited reduced NAA/Cr in the brain before treatment, suggestive of neuronal damage, and this was followed by an increase in NAA/Cr following initiation of ART, but a subsequent reduction in NAA/Cr after prolonged treatment and the expression of cognitive impairment. Collectively these findings suggest that ART likely does not facilitate complete restoration of functional systems to premorbid levels.

The second observation is in regard to the surface area sizes of the putamen and the caudate. Several previous studies have reported significant atrophy of the caudate among infected individuals, particularly among patients with dementia (Aylward et al., 1993, 1995; Stout et al., 1998). How- ever, not all studies have found relationships between HIV infection, the expression of cognitive dysfunction, and caudate volume (e.g., Di Sclafani et al., 1997). In the present study, we also did not observe significant reductions in caudate or putamen size at the group level, and this may reflect the general health of our cohort.

While some caution is warranted in terms of taking too much from our correlational findings, it is noteworthy that caudate size correlated with cognitive function on multiple measures, and the direction of the relationships was in the predicted direction for each association (i.e., lower size associated with poorer performance). Of interest, caudate size correlated most strongly with cognitive measures purportedly mediated by frontal-subcortical circuits, including Trail Making A and B, total learning across trials and the Stroop. By contrast, the area size of the putamen, which has anatomical connections to the motor cortex (Romanelli et al., 2005) correlated more strongly with symbol digit and grooved pegboard. These findings suggest a possible doubledissociation between the affected subcortical regions of the brain and performance on tests of higher-order thinking and psychomotor slowing in HIV.

It is important to recognize that several correlations were conducted, and stringent controls for these correlations would reduce the number of statistically significant relationships. We elected not to enforce these constraints given the modest size of the samples, but it is worth noting that adoption of a threshold less than .01 would not have changed the general direction of the findings with the exception of the correlations between putamen area size and performances on two motor-based tests. Yet in both cases the effect size for these correlations is large, and therefore the loss of significance reflects power rather than limited association between the variables. As such, while caution is warranted in interpreting the correlational analyses the results provide some support for the differential relationship between these two nuclei and the cognitive functions subserved by these neural systems in HIV.

Overall our results suggest that MRS changes are more prominent than quantitative MRI changes in the early stages of CNS involvement associated with HIV. However it is important to note that in the absence of longitudinal data, conclusions regarding the evolution of MRS $v s$. volumetric abnormalities in HIV remain speculative. With that caveat noted, our cross-sectional data suggests a model of CNS involvement in HIV characterized by early metabolic effects followed by changes in size within select subcortical gray matter regions with advanced disease burden. It is possible that cortical area changes also occur in the most advanced stages of disease progression. This is not to suggest that the cortical or subcortical regions are not impacted at a neuropathological level earlier in the process. Rather, these changes may not be detected by quantitative MRI until much later in the progression of the disease. Studies that examine cortical thinning on MRI in a healthy, treated sample would help define this issue more clearly.

Future studies are also needed to determine whether the expression of cognitive change and underlying MRI/MRS 


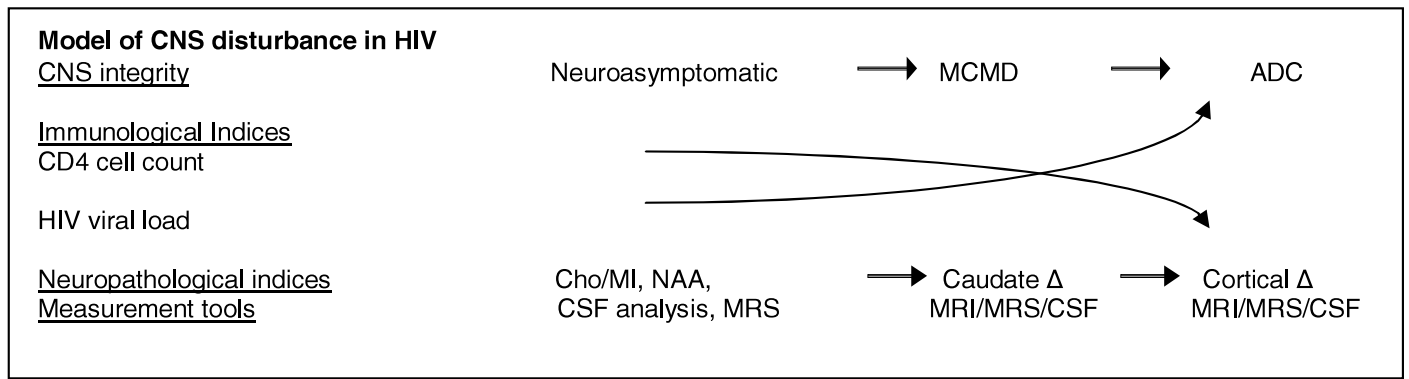

Fig. 1. Proposed model of the evolution of brain changes associated with HIV infection.

abnormalities evolves in a continuously linear manner as indicated in Figure 1, and what viral and host factors increase vulnerability to each stage in the process. Additional neuroimaging methods (e.g., functional MRI and DTI) may be particularly useful to accomplish these goals in future studies. As an example, Cloak et al. (2004) recently demonstrated significant diffusion abnormalities among HIV patients, and absolute MRS changes within these regions of diffusion changes. Similar approaches using multiple imaging methodologies may reveal the neurobiological signatures associated with each stage of HIV CNS involvement.

It is important to note, however, that a recent study has reported significant pathology in other regions of the brain outside the caudate and other basal ganglia structures, including the hippocampus (Moore et al., 2006). These latter findings are interesting because they emphasize that neuropathology in HIV is not restricted to the basal ganglia and the virus and associated inflammatory cascade may involve medial temporal lobe structures known to be important in cognitive operations. It is not clear, however, what cognitive skills/circuits are most impacted by these neuropathological findings, and/or whether the impact of these brain abnormalities is moderated by age. Recent studies of cognitive impairment in HIV suggest that the pattern of deficits largely remains "subcortical" in nature in this new era of chronic treatment (Lojek \& Bornstein, 2005) but this may change with chronicity of the disease and associated treatment as well as the advancing age of the HIV population.

\section{ACKNOWLEDGMENTS}

Supported by the AIDS Clinical Trials Group (NIAID), and grants NS36524 (NINDS), AI38855 (NIAID), MH60565 (NIMH), MH65857 (NIMH), and DA15045 (NIDA).

\section{REFERENCES}

Anonymous Army Individual Test Battery. Manual of directions and scoring. (1944). Washington, DC: War Department, Adjutant General's Office.

Aylward, E.H., Brettschneider, P.D., McArthur, J.C., Harris, G.J., Schlaepfer, T.E., Henderer, J.D., Barta, P.E., Tien, A.Y., \& Pearlson, G.D. (1995). Magnetic resonance imaging measurement of gray matter volume reductions in HIV dementia. American Journal of Psychiatry, 152, 987-994.

Aylward, E.H., Henderer, J.D., McArthur, J.C., Brettschneider, P.D, Harris, G.J., Barta, P.E, \& Pearlson, G.D. (1993). Reduced basal ganglia volume in HIV-1-associated dementia: Results from quantitative neuroimaging. Neurology, 43, 2099-2104.

Brew, B.J. (2004). Evidence for a change in AIDS dementia complex in the era of highly active antiretroviral therapy and the possibility of new forms of AIDS dementia complex. AIDS, 18, S75-S78.

Brickman, A.M., Buchsbaum, M.S., Shihabuddin, L., Hazlett, E.A., Borod, J.C., \& Mohs, R.C. (2003). Striatal size, glucose metabolic rate, and verbal learning in normal aging. Brain Research: Cognitive Brain Research, 17, 106-116.

Chang, L., Ernst, T., Leonido-Yee, M., Witt, M., Speck, O., Walot, I., \& Miller, E.N. (1999). Highly active antiretroviral therapy reverses brain metabolite abnormalities in mild HIV dementia. Neurology, 53, 782-789.

Chang, L., Ernst, T., Witt, M.D., Ames, N., Walot, I., Jovicich, J., DeSilva, M., Trivedi, N., Speck, O., \& Miller, E.N. (2003). Persistent brain abnormalities in antiretroviral-naive HIV patients 3 months after HAART. Antiviral Therapy, 8, $17-26$.

Chang, L., Lee, P.L., Yiannoutsos, C.T., Ernst, T., Marra, C.M., Richards, T., Kolson, D., Schifitto, G., Jarvik, J.G, Miller, E.N., Lenkinski, R., Gonzalez, G., \& Navia, B.A. (2004). HIV MRS Consortium. A multicenter in vivo proton-MRS study of HIVassociated dementia and its relationship to age. Neuroimage, 23, 1336-1347.

Cloak, C.C., Chang, L., \& Ernst, T. (2004). Increased frontal white matter diffusion is associated with glial metabolites and psychomotor slowing in HIV. Journal of Neuroimmunology, 157, 147-152.

Cohen, R.A., Boland, R., Paul, R., Tashima, K.T., Schoenbaum, E.E., Celentano, D.D., Schuman, P., Smith, D.K., \& Carpenter, C.C. (2001). Neurocognitive performance enhanced by highly active antiretroviral therapy in HIV-infected women. AIDS, 15, 341-345.

Comalli, P.E., Wapner, S., \& Werner, H. (1962). Interference effects of Stroop color-word test in childhood, adulthood, and aging. Journal of Genetics and Psychology, 100, 47-53.

Cummings, J. (1993). Frontal-subcortical circuits and human behavior. Archives of Neurology, 50, 873-880.

Dal Pan, G.J., McArthur, J.H., Aylward, E., Selnes, O.A., NanceSpronson, T.E., Kumar, A.J., Mellits, E.D., \& McArthur, J.C. (1992). Patterns of cerebral atrophy in HIV-1 infected individuals. Results of a quantitative MRI analysis. Neurology, 42, 2125-2130. 
Di Sclafani, V., Mackay, S., Meyerhoff, D.J., Norman, D., Weiner, M.W., \& Fein, G. (1997). Brain atrophy in HIV infection is more strongly associated with CDC clinical stage than with cognitive impairment. Journal of the International Neuropsychological Society, 3, 276-287.

Grant, I., Atkinson, J.H., Hesselink, J.R., Kennedy, C.J., Richman, D.D., Spector, S.A., \& McCutchan, J.A. (1987). Evidence for early central nervous system involvement in the acquired immunodeficiency syndrome (AIDS) and other human immunodeficiency virus (HIV) infections. Studies with neuropsychologic testing and magnetic resonance imaging. Annals of Internal Medicine, 107, 828-836.

Heaton, R.K., Marcotte, T.D., Mindt, M.R., Sadek, J., Moore, D.J., Bentley, H., McCutchan, J.A., Reicks, C., Grant, I., \& The HNRC Group. (2004). The impact of HIV-associated neuropsychological impairment on everyday functioning. Journal of the International Neuropsychological Society, 10, 317-331.

Hestad, K., McArthur, J.H., Dal Pan, G.J., Selnes, O.A., NanceSproson, T.E., Aylward, E., Mathews, V.P., \& McArthur, J.C. (1993). Regional brain atrophy in HIV-1 infection: Association with specific neuropsychological test performance. Acta Neurologica Scandinavica, 88, 112-118.

Klove, H. (1963). Clinical neuropsychology. In P.M. Forster (Ed.), The medical clinics of North America. New York: WB Saunders.

Lee, P.L., Yiannoutsos, C.T., Ernst, T., Chang, L., Marra, C.M., Jarvik, J.G., Richards, T.L., Kwok, E.W., Kolson, D.L., Simpson, D., Tang, C.Y., Schifitto, G., Ketonen, L.M., Meyerhoff, D.J., Lenkinski, R.E., Gonzalez, R.G., \& Navia, B.A. (2003). HIV MRS Consortium. A multi-center 1H MRS study of the AIDS dementia complex: Validation and preliminary analysis. Journal of Magnetic Resonance Imaging, 17, 625-633.

Lojek, E. \& Bornstein, R.A. (2005). The stability of neurocognitive patterns in HIV infected men: Classification considerations. Journal of Clinical and Experimental Neuropsychology, 27, 665-682.

Marcotte, T.D., Wolfson, T., Rosenthal, T.J., Heaton, R.K., Gonzalez, R., Ellis, R.J., Grant, I. \& HIV Neurobehavioral Research Center Group. (2004). A multimodal assessment of driving performance in HIV infection. Neurology, 63, 1417-1422.

Meyerhoff, D J., Bloomer, C., Cardenas, V., Norman, D., Weiner, M.W., \& Fein, G. (1999). Elevated subcortical choline metabolites in cognitively and clinically asymptomatic HIV + patients. Neurology, 52, 995-1003.

Miller, E.N. (1990). California Computerized Assessment Package (CalCAP). Los Angeles: Norland Software.

Miller, E.N., Satz, P., \& Visscher, B.V. (1991). Computerized and conventional neuropsychological assessment of HIV-1 infected homosexual men. Neurology, 41, 1608-1616.

Miller, E.N., Selnes, O.A., McArthur, J.C., Satz, P., Becker, J.T., Cohen, B.A., Sheridan, K., Machado, A.M., Van Gorp, W.G., \& Visscher, B. (1990). Neuropsychological performance in HIV1-infected homosexual men: The Multicenter AIDS Cohort Study (MACS). Neurology, 40, 197-203.

Moore, D.J., Masliah, E., Rippeth, J.D., Gonzalez, R., Carey, C.L., Cherner, M., Ellis, R.J., Achim, C.L., Marcotte, T.D., Heaton, R.K., Grant, I., \& HNRC Group. (2006). Cortical and subcortical neurodegeneration is associated with HIV neurocognitive impairment. AIDS, 20, 879-887.

Navia, B.A. (1997). Clinical and biologic features of the AIDS dementia complex. Neuroimaging Clinics of North America, 197, 581-592.
Navia, B.A. \& Gonzalez, R.G. (1997). Functional imaging of the AIDS dementia complex and the metabolic pathology of the HIV-1-infected brain. Neuroimaging Clinics of North America, 197, 431-445.

Navia, B.A. \& Rostasy, K. (2005). The AIDS dementia complex: Clinical and basic neuroscience with implications for novel molecular therapies. Neurotoxicology Research, 8, 3-24.

Neuenburg, J.K., Brodt, H.R., Herndier, B.G., Bickel, M., Bacchetti, P., Price, R., Grant, R.M., \& Schlote, W. (2002). HIVrelated neuropathology, 1985 to 1999: Rising prevalence of HIV encephalopathy in the era of highly active antiretroviral therapy. Journal of Acquired Immune Deficiency Syndromes, 31, 171-177.

Osowiecki, D.M., Cohen, R.A., Morrow, K.M., Paul, R.H., Carpenter, C.C., Flanigan, T., \& Boland, R.J. (2000). Neurocognitive and psychological contributions to quality of life in HIV1-infected women. AIDS, 14, 1327-1332.

Paul, R., Cohen, R., Navia, B., \& Tashima, K. (2002). Relationships between cognition and structural neuroimaging findings in adults with human immunodeficiency virus type-1. Neuroscience and Biobehavioral Review, 26, 353-359.

Paul, R.H., Yiannoutsos, C.Y., Miller, E.N., Chang, L., Marra, C.M., Schifitto, G., Ernst, T., Singer, E., Richards, T., Jarvik J., Price, R., Myerhoff, D.J., Kolson, D., Ellis, R.J., Gonzalez, G., Lenkinski, R.E., Cohen, R.A., \& Navia, B.A, for the ACTG 301 and 700 teams and the HIV MRS Consortium. (2007). Proton MRS and neuropsychological correlates in AIDS dementia complex: Evidence of subcortical specificity. The Journal of Neuropsychiatry and Clinical Neurosciences, 19, 283-292.

Rey, A. (1964). L'examen clinique en psychologie. Paris: Presses Universitaires de France.

Robertson, K.R., Robertson, W.T., Ford, S., Watson, D., Fiscus, S., Harp, A.G., \& Hall, C.D. (2004). Highly active antiretroviral therapy improves neurocognitive functioning. Journal of Acquired Immune Deficiency Syndromes, 36, 562-566.

Romanelli, P., Esposito, V., Schaal, D.W., \& Heit, G. (2005). Somatotopy in the basal ganglia: Experimental and clinical evidence for segregated sensorimotor channels. Brain Research: Brain Research Review, 48, 112-128.

Sacktor, N.C., Lyles, R.H., Skolasky, R.L., Anderson, D.E., McArthur, J.C., McFarlane, G., Selnes, O.A., Becker, J.T., Cohen, B., Wesch, J., \& Miller, E.N. (1999). Combination antiretroviral therapy improves psychomotor speed performance in HIV-seropositive homosexual men. Multicenter AIDS Cohort Study (MACS). Neurology, 52, 1640-1647.

Sacktor, N., Tarwater, P.M., Skolasky, R.L., McArthur, J.C., Selnes, O.A., Becker, J., Cohen, B., Miller, E.N., \& Multicenter for AIDS Cohort Study (MACS). (2001). CSF antiretroviral drug penetrance and the treatment of HIV-associated psychomotor slowing. Neurology, 57, 542-544.

Selnes, O.A., Galai, N., Bacellar, H., Miller, E.N., Becker, J.T., Wesch, J., Van Gorp, W., \& McArthur, J.C. (1995). Cognitive performance after progression to AIDS: A longitudinal study from the Multicenter AIDS Cohort Study. Neurology, 45, 267-275.

Smith, A. (1973). Symbol Digit Modalities Test Manual. Los Angeles: Western Psychological Services.

Stout, J.C., Ellis, R.J., Jernigan, T.L., Archibald, S.L., Abramson, I., Wolfson, T., McCutchan, J.A., Wallace, M.R., Atkinson, J.H., \& Grant, I. (1998). Progressive cerebral volume loss in human immunodeficiency virus infection: A longitudinal volumetric magnetic resonance imaging study. HIV Neurobehavioral Research Center Group. Archives of Neurology, 55, 161-168. 
Stroop, J.R. (1935). Studies of interference in serial verbal reaction. Journal of Experimental Psychology, 18, 643-662.

Tarasow, E., Wiercinska-Drapalo, A., Kubas, B., Dzienis, W., Orzechowska-Bobkiewicz, A., Prokopowicz, D., \& Walecki, J. (2003). Cerebral MR spectroscopy in neurologically asymptomatic HIV-infected patients. Acta Radiologica, 44, 206-212.

Tucker, K.A., Robertson, K.R., Lin, W., Smith, J.K., An, H., Chen, Y., Aylward, S.R., \& Hall, C.D. (2004). Neuroimaging in human immunodeficiency virus infection. Journal of Neuroimmunology, 157, 153-162.

Wilkinson, I.D., Lunn, S., Miszkiel, K.A., Miller, R.F., Paley, M.N., Williams, I., Chinn, R.J., Hall-Craggs, M.A., Newman, S.P.,
Kendall, B.E., \& Harrison, M.J. (1997). Proton MRS and quantitative MRI assessment of the short-term response to antiretroviral therapy in AIDS. Journal of Neurology, Neurosurgery, and Psychiatry, 63, 477-482.

Yiannoutsos, C.T., Ernst, T., Chang, L., Lee, P.L., Richards, T., Marra, C.M., Meyerhoff, D.J., Jarvik, J.G., Kolson, D., Schifitto, G., Ellis, R.J., Swindells, S., Simpson, D.M., Miller, E.N., Gonzalez, R.G., \& Navia, B.A. (2004). Regional patterns of brain metabolites in AIDS dementia complex. Neuroimage, 23, 928-935. 